\title{
Application of ART to Time-coded Emission Tomography
}

\author{
KENNETH F. KORAL, PH.D. and W. LESLIE ROGERS, PH.D. \\ R-3054 Kresge II, University of Michigan Medical Center, Ann Arbor, \\ MI 48109, U.S.A.
}

Received 20 June 1978, in final form 14 March 1979

\begin{abstract}
Devices for single-photon emission tomography currently take projections either in a plane over a full angular range $\left(0-360^{\circ}\right)$ or in a volume with a limited angular range. The planar, pseudo-random, time-coded aperture, in conjunction with an Anger camera, is a device of the limited angular range type. It employs multiple pinholes whose transmission varies as a function of time. Previously, image reconstruction was accomplished by simple back-projection of coefficients obtained by time-correlating pinhole transmission with detector-element count rate, resulting in a low-contrast image.

Using the Algebraic Reconstruction Technique (ART) a method is introduced for division of the correlation coefficients into subsets allowing the three-dimensional reconstruction to be accomplished on a minicomputer. Results from simulations and experimental phantom data show that ART improves depth resolution compared to back-projection, that under-relaxation produces better images in the case of noisy data, and that the division of the correlation coefficients into subsets has no effect on quelity. The images depict the expected resolution degradation in the direction normal to the detector plane due to the limited angular range of projections but yield quantitative results whose relative values are good, even though attenuation is neglected.
\end{abstract}

\section{Introduction}

Tomographic imaging in nuclear medicine using gamma-emitting radiotracers is currently under active investigation. A planar, multi-pinhole, pseudo-random, time-coded aperture in conjunction with an Anger camera (Koral, Knoll and Rogers 1977) is a tomographic device that operates in a fixed position and covers a limited angular range. This aperture has already been shown to have better resolution than that of a parallel hole collimator in the case of moderately sized objects, due to image magnification onto the camera. It has also been shown to have higher efficiency than a single pinhole of the same resolution when imaging planar objects (Koral, Rogers and Knoll 1975). Finally, the limited tomography of this device is sufficient to improve significantly the contrast of cold nodules in thyroid imaging compared to multiple view pinhole imaging (Koral, Freitas, Rogers and Keyes 1979).

In comparison with tomographic devices utilising parallel hole collimation and rotation (Budinger and Gullberg 1974, Kuhl, Edwards, Ricci, Yacob, Mich and Alavi 1976, Keyes, Orlandea, Heetderks, Leonard and Rogers 1977), the coded aperture has these advantages: (1) the system is mechanically simple, (2) the aperture can be placed directly over and near a small organ, and (3) the longitudinal resolution, that in a plane parallel to the camera face, is very good. A new fixed-position 7-pinhole collimator (Vogel, Kirch, LeFree, Klingensmith, Trow and Steele 1977, Vogel, Kirch, LeFree and Steele 1978) is similar to the 
time-coded aperture but does not code the data and has the disadvantage of gross sampling over the angular range of projections (however, this gross sampling does reduce the reconstruction time). The disadvantage of either of these fixed-position systems in comparison to a system involving rotation is that a complete set of projections $\left(0\right.$ to $\left.360^{\circ}\right)$ is not obtained. Compared to a two-dimensional reconstruction, a true three-dimensional reconstruction has been shown (Colsher 1976) partially to compensate for this limited angular range, but one must as yet accept a transverse resolution which is inferior to the longitudinal resolution.

A disadvantage of the time-coded aperture is the long reconstruction time. Routine clinical application will require implementation of the algorithm outlined herein on a digital microprocessor which emphasises parallel computation. However, this microprocessor might have other uses or might be part of an existing system.

A brief description of time-coded tomography follows. The collimator is similar to a standard pinhole collimator but with a large square opening or defining aperture in place of the pinhole. Over this defining aperture lies a long lead plate with multiple pinholes arranged on a unit grid. Data are taken in $L$ distinct time intervals, between each of which the code plate is translated one unit in its long direction. As the plate translates, each positional element in the defining aperture is subject to a series of conditions of either complete transmission or complete absorption of impinging gamma rays, according to a known code. A time correlation of aperture-element transmission and detector-element count rate is then carried out for each aperture element in combination with each detector element. Previously, image reconstruction was carried out by simple back-projection of these time correlations into the image volume with the result being a low-contrast image.

It is shown in section 2 below that the time-coded-aperture reconstruction problem is amenable to ART, the Algebraic Reconstruction Technique (Herman, Lent and Rowland 1973, Herman and Lent 1976) and so image improvement can be expected. Since a true three-dimensional reconstruction is to be carried out, to compensate for the limited angular range, the ART computation could be prohibitively long and require extensive computer storage but it is here accomplished on a minicomputer by employing a special image-volume geometry and a core-saving division of the data into subsets. No correction for attenuation is carried out in the present formulation. In section 3 , the results from one particular coded aperture and several variations of ART are considered. The effects of array thinning, relaxation parameter and mode of sampling together with the influence of both noise and spatial distortion are examined by means of two-dimensional simulation. Both quantitative and qualitative results are then given for experimentally obtained phantom data.

\section{Theory}

\subsection{Correlation coefficient as projection element}

Time-coded tomography involves a two-step reconstruction in which one first calculates the mean subtracted correlation coefficient for the $i$ th detector 
and the $j$ th aperture element over $\nu$ time intervals, $Q_{i j}$ :

$$
Q_{i j}=\sum_{\nu=1}^{L} C_{\nu i} T_{j+\nu}-m \sum_{\nu=1}^{L} C_{\nu i}
$$

where $C_{\nu i}$ is the number of counts in the $i$ th detector during the $\nu$ th time interval, $T_{j+\nu}$ is $(j+\nu)$ th element of a pseudo-random code sequence and $m$ is the mean transmittance of the code. To do the second step in the reconstruction, define the line from the centre of the $i$ th detector through the centre of the $j$ th aperture as the $i j$ th ray. Then consider $N_{\mathrm{p}}$ discrete depths indexed by integer $k$ and define the image strength per unit area in the $k$ th longitudinal plane at the point intersected by the $i j$ th ray as $I_{l k}, l=f(i, j, k)$. Assuming a slowly varying image, we then show in Appendix 1 that a correlation coefficient corrected for finite geometry effects, $\tilde{Q}_{i j}$, is equal to the simple ray sum of image strength/unit area over $N_{\mathrm{p}}$ planes:

$$
\tilde{Q}_{i j}=\sum_{k=k i}^{k} I_{l k}, \quad l=f(i, j, k)
$$

where $N_{\mathrm{p}}=k_{f}-k_{i}+1$.

The corrected correlation coefficient, $\tilde{Q}_{i j}$, is, therefore, a projection element for the image strength per unit area with the angle of the projection defined by the ij subscripts.

The correction for finite geometry effects is made as follows:

$$
\bar{Q}_{i j}=Q_{i j} /\left(F S_{i j} \cos ^{3} \theta_{i j}\right)
$$

with $F$ equal to a constant depending on the code and geometrical factors, $S_{i j}$ equal to the open area of the $j$ th aperture as viewed by the $i$ th detector, and $\theta_{i j}$ equal to the angle between the normal and the $i j$ th ray. This correction is seen to involve boosting the value of those correlation coefficients which view the source at a large angle so as to preserve consistency with the image. The open area $S_{i j}$ is given by a geometrical formula:

$$
S_{i j}=\pi\left(a-t \tan \theta_{i j}\right)\left(a^{2}-t^{2} \tan ^{2} \theta_{i j}\right)^{\frac{1}{2}} / 4
$$

where $t$ is the thickness of the code plate and $a$ is the diameter of the pinholes. In practice, because of edge penetration, $t$ is usually set to an empirical value derived from requiring agreement between eqn (4) and the image of a field flood taken with a single pinhole.

\subsection{Image space}

To save computing time, the image volume is divided into elements such that all element centres lie along rays (fig. $l(a)$ ). The development below is for one longitudinal direction in combination with depth but holds for both orthogonal longitudinal directions. The division is accomplished by first requiring that rays which pass through a given aperture element and adjacent detector elements pass through adjacent image elements in all planes as depicted in fig. $1(b)$ :

$$
g_{k} / d=A_{k} / B
$$




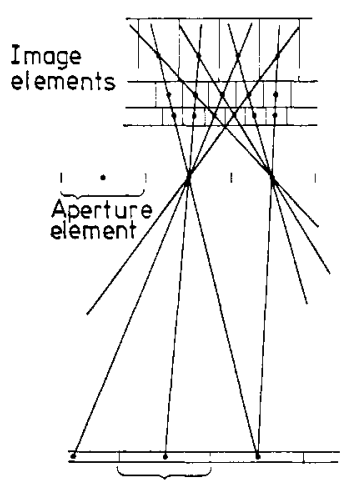

Detector element

(a)

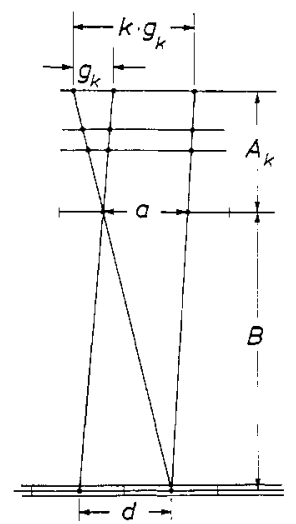

(b)

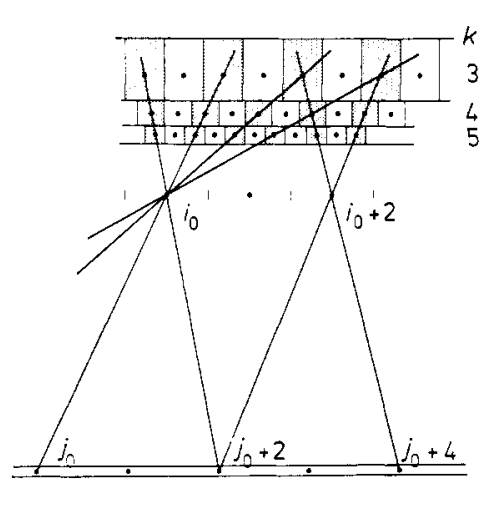

(c)

Fig. 1. Division of image volume into elements such that each ray passes through the centre of one element at each depth. (a) Unthinned algorithms; all image elements are referenced. (b) Geometry involved in establishing the mean depths, $A_{k}$, and image element dimensions, $g_{k}$. (c) Thinned algorithm-one subset of coefficients (detector $j_{0}, j_{0}+2, \ldots$ in correlation with aperture $i_{0}, i_{0}+2, \ldots$ ) references only a thinned image (shown shaded). This would not be the case with a subset such as $j_{0}, j_{0}+2 \ldots$ and $i_{0}, i_{0}+1 \ldots$.

Secondly, rays originating from a given detector element and passing through adjacent aperture elements pass through image elements separated by $k-1$ elements in the $k$ th plane:

$$
k g_{k} / a=\left(A_{k}+B\right) / B
$$

The integer relationships expressed in eqns (5) and (6) determine the required plane depths, $A_{k}$, and image element dimensions, $g_{k}$ :

$$
\begin{aligned}
A_{k} & =a B /(k d-a) \\
g_{k} & =a d /(k d-a), \quad k=1,2,3, \ldots .
\end{aligned}
$$

\subsection{Algebraic Reconstruction Technique (ART)}

The entire set of linear equations to which (2) belongs can be represented as:

$$
\tilde{\mathbf{Q}}=\mathbf{W} \mathbf{I}
$$

where $\tilde{\mathbf{Q}}$ is a column vector of dimension $m=N_{\mathrm{D}} \times N_{\mathrm{A}}$, I is a column vector of dimension $n=N_{\mathrm{I}} \times N_{\mathrm{p}}$ and $\mathrm{W}$ is an $m$ by $n$ matrix of weights $\left(N_{\mathrm{D}}, N_{\mathrm{A}}, N_{\mathrm{I}}\right.$ and $N_{\mathrm{p}}$ are the number of detectors, apertures, image elements and planes respectively). The matrix $\mathbf{W}$ is sparse and for point sampling, the use of a single image element in each plane for each ray, is composed of only l's and 0's. The method used for solution of these equations in this paper is the partially constrained Algebraic Reconstruction Technique (ART).

The form of this iterative technique appropriate to $0-1$ weights is

$$
\begin{gathered}
\Delta=\tilde{Q}_{i j}-\sum_{k=k_{i}}^{k_{j}} I_{l k}^{\rho}, \quad l=f(i, j, k) \\
I_{l k}^{\rho+1}=\max \left[\left(I_{l k}^{\rho}+r \Delta / N_{\mathrm{p}}\right), 0\right], \quad k=k_{i}, \ldots, k_{f}
\end{gathered}
$$


where $\Delta$ is the discrepancy between projection element and ray sum at the $\rho$ th iteration, $I_{l k}^{\rho}$ is the $l k$ th image element strength at the $\rho$ th iteration, $r$ is a relaxation parameter and $\max (a, 0)$ is an operator which returns the value $a$ if $a$ is positive and zero otherwise. The starting condition is $I_{l k}^{0}=0$ for all $l k$ and the order is to step systematically through the aperture subscript faster than the detector subscript.

An algorithm variation, ART2, and a criterion for stopping the iteration process (Herman et al. 1973) are investigated in section 3. In ART2 the max operation is eliminated from eqn (10) and inserted into eqn (9) as follows:

$$
\begin{gathered}
\Delta=\widetilde{Q}_{i j}-\sum_{k=k_{i}}^{k_{f}} \max \left(I_{l k}^{\rho}, 0\right) \\
I_{l k}^{\rho+1}=I_{l k}^{\rho}+r \Delta / N_{\mathrm{p}}, \quad k=k_{i}, \ldots, k_{f} .
\end{gathered}
$$

After the last iteration, the max operation is applied to all image elements to set negative values to 0 . The convergence criterion is to stop when the variance from the mean of the images changes by less than a fixed percentage between iterations. That is:

$$
\begin{gathered}
M^{\rho}=\sum_{l, k} I_{l, k}^{\rho} / N \\
V^{\rho}=\sum_{l, k}\left(I_{l k}^{\rho}-M^{\rho}\right)^{2} / N \\
V^{\rho+1}-V^{\rho} \leqslant P V^{\rho}
\end{gathered}
$$

where $M^{\rho}$ is the mean and $V^{\rho}$ is the variance after $\rho$ iterations, $N$ is the total number of elements in the image volume, and $P$ is the stopping fraction, usually $4 \%$ in the work detailed here.

\subsection{Thinning}

For the coded aperture which is used in this study (see section 3.1), the imageelement longitudinal dimension, $g_{k}$, is as small as $0.6 \mathrm{~mm}$ while the corresponding field of view is a $7.8 \mathrm{~cm}$ square. Thus, a $192 \times 192$ matrix is required for this plane and a very large computer core is required for the total image.

The large number of image elements can be reduced by dividing the correlation data into subsets, for each of which the detector and aperture arrays are thinned, so that only a thinned version of the image array is required. Fig. $1(c)$ shows a data subset for a thinning factor, $h$, of 2 -that is, only data corresponding to every second detector and every second aperture is considered. Because of the integer relationships imposed by eqns (5) and (6), only a subset of the image array, every second image element in each plane, is referenced. Indexing the starting aperture element by one while using the same detector elements (and vice versa) produces a similar sub-array in image space. (Note: specific sub-arrays contain some elements in common.) For two-dimensional detector and aperture arrays, the total number of subsets is $h^{4}$ and the computer storage requirement is cut by a factor of $h^{2}$. The final image averages the independent values obtained for each image element. If the image grid is 
fine with respect to the total resolution, small elements can be combined, $h^{2}$ to 1 , which reduces computer disc storage and simplifies the averaging.

In general, the set of equations given by eqn (8) is overdetermined $(m>n)$ if all the equations are independent. In thinning, the number of equations decreases faster than the number of image elements so that each subset can be guaranteed to be underdetermined. Effects of this fact are looked for in the simulations of section 3.2 .

\subsection{Integral sampling}

Instead of point sampling the image-strength density, one can average over several image elements, the number to be determined by the finite size of the backprojected cone:

$$
\tilde{Q}_{i_{3}}=\sum_{l=l_{i}}^{l_{j}} \sum_{k=k_{i}}^{k_{f}} w_{l k} I_{l k}
$$

where the $u_{l k}$ are fractional weights. In this case, the general ART formulation (Herman et al. 1973) is used and the calculation is longer since a second sum is involved and more image elements need to be corrected for each correlation coefficient.

\section{Methods and results}

\subsection{Experimental dimensions and computation times}

The aperture used for the experimental investigation is an $11 \times 11$ array of contiguous elements $3.57 \mathrm{~mm}$ in diameter (Koral et al. 1975). It is spaced $15 \mathrm{~cm}$ from an Anger camera detector which is $25.4 \mathrm{~cm}$ in diameter with a $64 \times 64$ digitisation. These dimensions yield the values given in table 1 for the depths

Table 1. Dimensions of image space

\begin{tabular}{|c|c|c|c|c|c|c|}
\hline \multirow[b]{2}{*}{$\begin{array}{c}\text { Plane } \\
\text { index, } \\
k\end{array}$} & \multirow[b]{2}{*}{$\begin{array}{l}\text { Aperture } \\
\text { to plane } \\
\text { distance, } \\
A_{k}(\mathrm{~cm})\end{array}$} & \multirow[b]{2}{*}{$\begin{array}{c}\text { Fine-grid } \\
\text { image- } \\
\text { element size, } \\
g_{k}(\mathrm{~mm})\end{array}$} & \multirow[b]{2}{*}{$\begin{array}{c}\text { Coarse-grid } \\
h=3 \text { image- } \\
\text { element size, } \\
g_{k}(\mathrm{~mm})\end{array}$} & \multirow[b]{2}{*}{$\begin{array}{c}\text { Thickness of } \\
\text { longitudinal } \\
\text { plane, } \\
t_{k}(\mathrm{~cm})\end{array}$} & \multicolumn{2}{|c|}{ Size of square field of view } \\
\hline & & & & & $\begin{array}{l}\text { In fine-grid } \\
\text { image } \\
\text { elements }\end{array}$ & $(\mathrm{cm})$ \\
\hline 7 & $2 \cdot 2$ & 0.59 & 1.75 & $0 \cdot 36$ & 134 & $7 \cdot 85$ \\
\hline 6 & $2 \cdot 65$ & 0.70 & $2 \cdot 10$ & 0.52 & 124 & $8 \cdot 68$ \\
\hline 5 & $3 \cdot 3$ & 0.87 & $2 \cdot 61$ & 0.82 & 114 & 9.93 \\
\hline 4 & $4 \cdot 35$ & $1 \cdot 15$ & $3 \cdot 45$ & $1 \cdot 44$ & 104 & 11.98 \\
\hline 3 & $6 \cdot 4$ & 1.70 & $5 \cdot 10$ & $3 \cdot 20$ & 94 & 15.99 \\
\hline
\end{tabular}

and scale factors of the reconstructed planes. The angular range of projections for a central point in the $k=5$ plane $(3.3 \mathrm{~cm}$ depth $)$ is then computed to be that over a cone with half angle $28^{\circ}$.

Calculations are carried out on a $32 \mathrm{~K}$ minicomputer with the following running times. Computation of the correlation coefficients with a non-optimised assembly language program and correction for angular variation with a similarly non-optimised Fortran program take $20 \mathrm{~min}$ for each program. 
Reconstruction of 4 planes by an 81-subset ART using a Fortran program which employs an assembly language subroutine for the inner loop takes one hour per iteration.

\subsection{Simulations}

Simulations in two dimensions (longitudinal distance and depth) are carried out with an 11-element aperture and an infinite detector with the element sizes and the depths of the 'planes' the same as in the experimental case. Since a signal-to-noise analysis is not carried out, the calculation is simplified by assuming a moving pinhole (code 10000000000 ) rather than a more general code and a perfect angular variation correction. The approximation of lumping the object-element strength into the centre of the element in generating the data is also made.

Fig. 2 presents the simulation results for a noise-free point source in the $2.2 \mathrm{~cm}$ plane as shown at the left. The ART reconstruction without thinning is essentially perfect after 5 iterations, allowing for the resolution of the finitesized aperture elements. This result justifies point sampling. The ART reconstruction with thinning is displayed in the next column. The thinning factor,

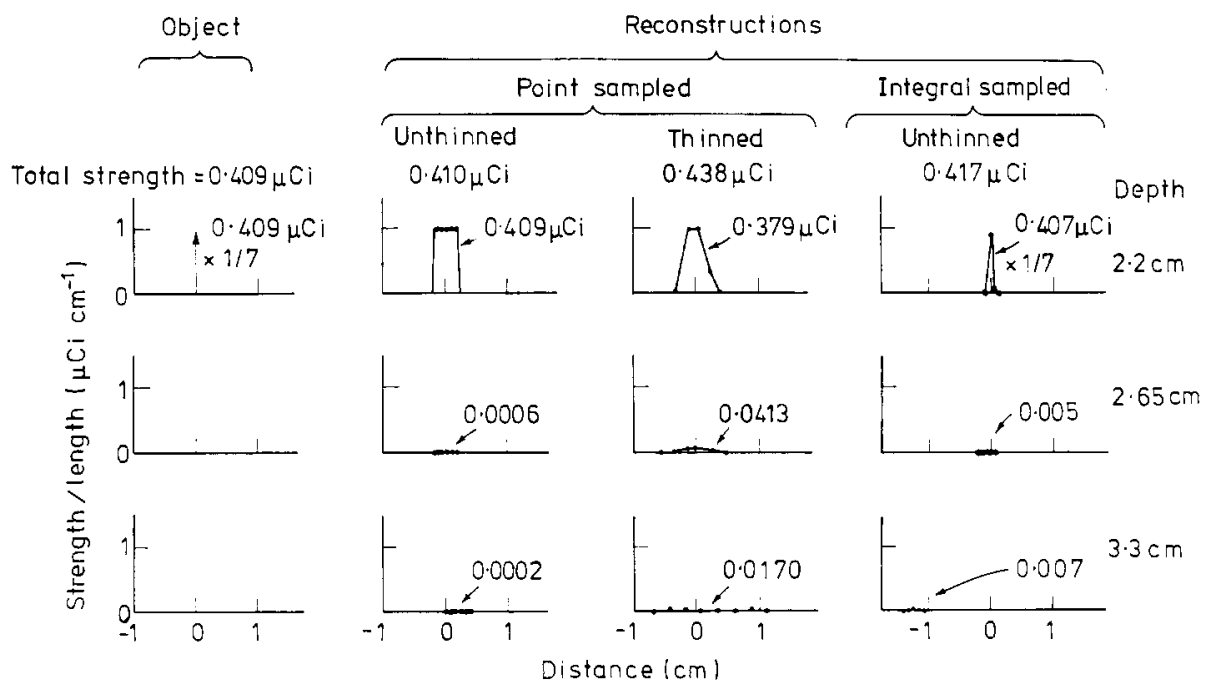

Fig. 2. Simulation results for a noise-free point source after 5 iterations of ART. The mean depth, $A_{k}$, of the longitudinal, one-dimensional 'planes' is given at the right. Depth resolution is very good for this simple object.

$h$, equals 3 as it will throughout the rest of this paper and the image elements are combined 3 to 1 and displayed on a coarse grid. There is an artificial drop-off in the $2.2 \mathrm{~cm}$ image due to the registration of the coarse and fine grid and there is some residual strength in the other two planes. With more iterations, this strength decreases further and so the thinning technique has little effect. The integral-sampled reconstruction without thinning at the right shows a 
much sharpened image in the $2.2 \mathrm{~cm}$ plane, implying that this sampling produces 'super resolution".

Fig. 3 shows noise-free simulation results for a source volume which is more nearly filled and which is, therefore, a more difficult reconstruction problem. The ART reconstruction without thinning and with relaxation parameter equal to 1 produces an image which has the correct total strength $(25 \mu \mathrm{Ci})$ but which

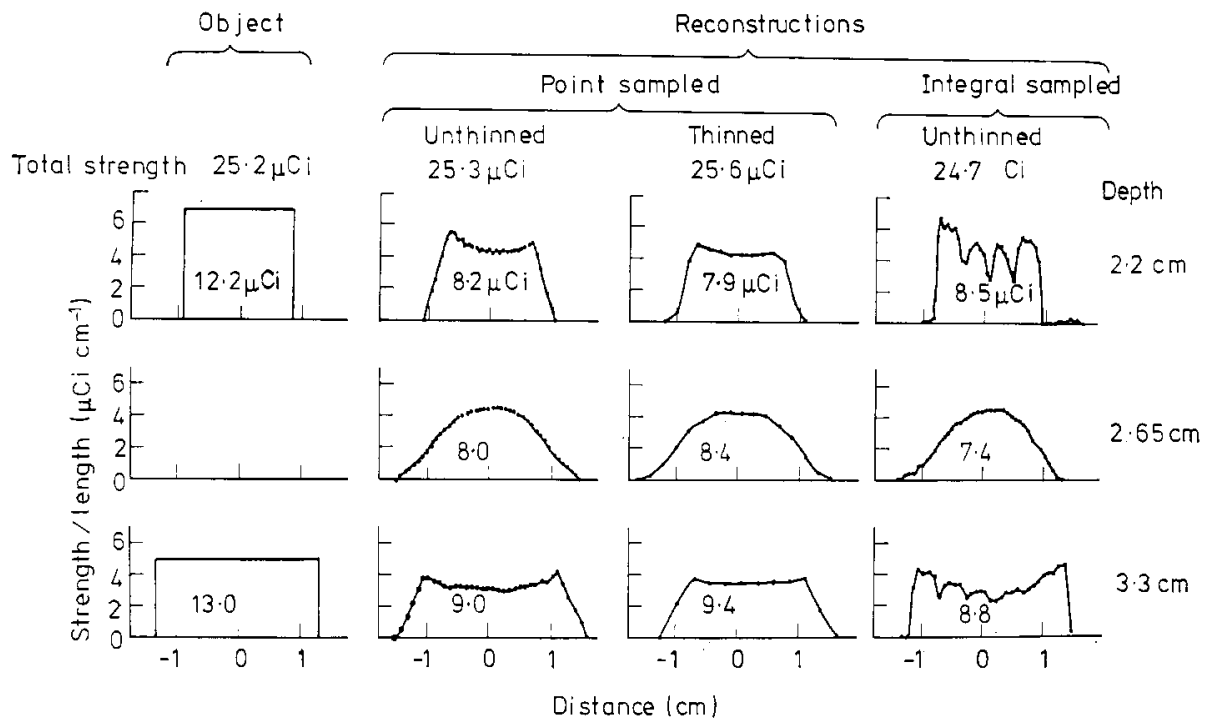

Fig. 3. Simulation results after 5 iterations of ART for two line sources located behmia each other. For this object part of the correctly reconstructed total strength appears in the $2.65 \mathrm{~cm}$ 'plane' because of poor depth resolution.

has the relatively poor resolution in depth one expects. There is also some overshoot in the shapes of the reconstructed lines. The result with the thinned algorithm, $r=1$, and a coarse display grid is similar and perhaps slightly improved as there is less overshoot. If one employs a relaxation parameter of $1 / 8$ instead of 1 , the images, which are not shown, are similar but 10 iterations are required to reach the total strength of $25 \mu \mathrm{Ci}$.

The images as reconstructed by the unthinned-array algorithm with integral sampling are shown at the right. The total strength in the empty 'plane' is reduced and the objects are again rendered with better resolution at the edges but these favourable characteristics are offset by a low-frequency ringing. Because of this ringing, integral sampling is not investigated further in this paper.

Reconstructions of the two-line-source object of fig. 3 with noise and with noise plus a camera distortion which mimics Anger camera edge packing are illustrated in fig. 4. For a typical time interval, the effect of Poisson statistics upon the number of counts in each detector element is shown at the lower left (the line being noise-free data). The thinned and unthinned algorithm with relaxation parameter equal to 1 produce very similar results, indicating that 


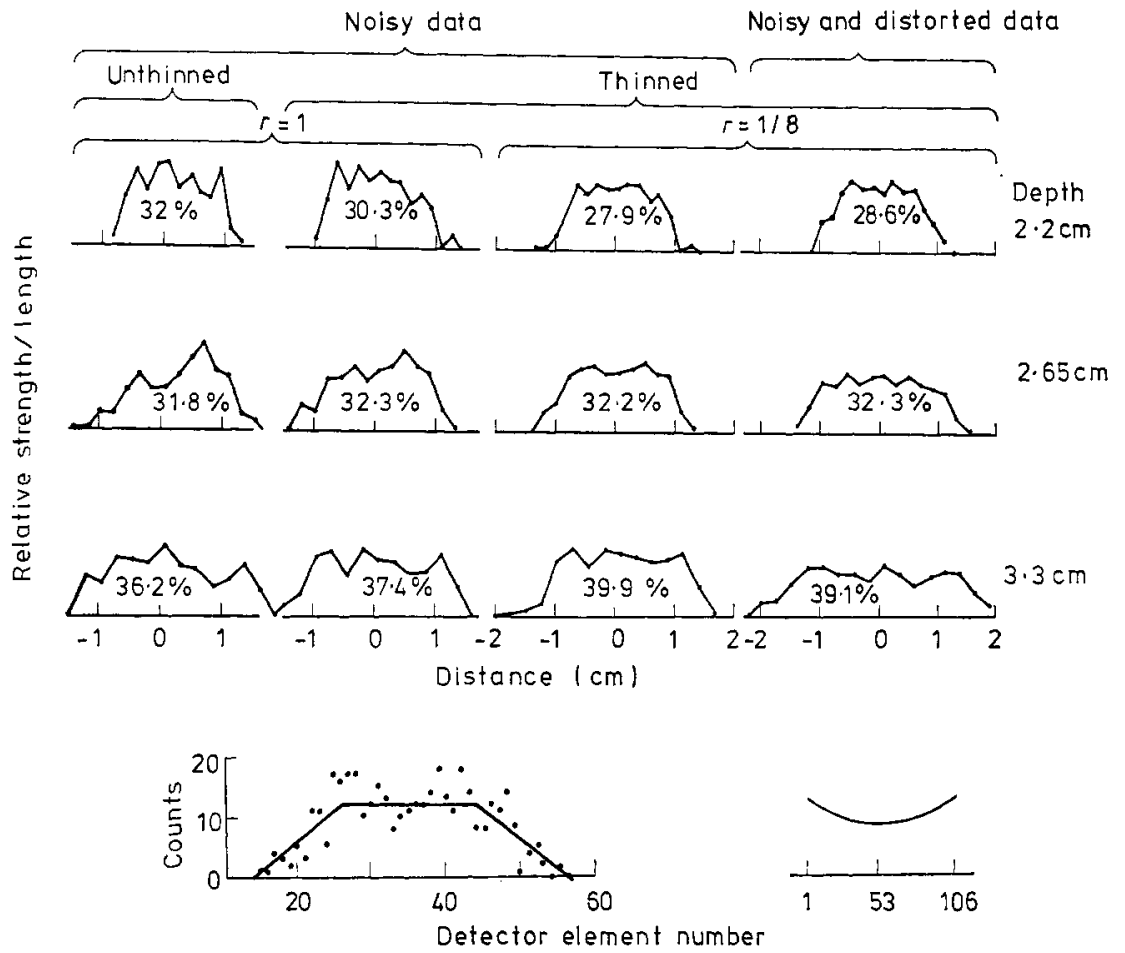

Fig. 4. Simulation results for the object of fig. 3 when noise or noise and camera distortion are taken into account. With noise alone, the unthinned and thinned algorithm produce similar results as shown for $r=1$ in the left two columns. With noise alone, $r=1 / 8$ produces smoother images than $r=1$ as seen by comparing the middle two columns. The magnitude of noise is shown at the lower left. With noise and distortion the back 'plane' is considerably broadened as seen by comparing the right two columns. The magnitude of distortion is demonstrated by the curvature of the plot at the lower right.

even with noise thinning has little effect. A significant improvement in the uniformity of the results occurs for both algorithms when a relaxation parameter of $1 / 8$ is employed as is shown for the thinned algorithm in the third column of the figure.

The assumed camera distortion tends to misplace counts towards the edges; the magnitude of the distortion is illustrated at the lower right by the results for a field-flood input. At the top right are the image reconstructions with the thinned algorithm and a relaxation parameter of $1 / 8$. The image in the deepest plane has been considerably broadened, but otherwise the images have not been drastically affected.

\subsection{Phantoms}

Experimental results for data obtained from phantoms filled with ${ }^{99} \mathrm{Tc}^{\mathrm{m}}$ and reconstructed by the thinned algorithm are presented below. A point source located on axis at a depth of $2 \cdot 2 \mathrm{~cm}$ is reconstructed with ART2. Iteration for each subset of correlation coefficients is terminated automatically when the 
variance from the mean changes by less than $4 \%$, but this termination results in a change from a fixed limit of 4 iterations in only 4 of 81 subsets and in these only to 5 iterations. The result only approaches that for the simulated point source due to statistical noise, camera distortion and inaccuracies in the correction for finite geometry. However, the resolution is $3.5 \mathrm{~mm}$ FWHM and the peak image strength in the $2.65 \mathrm{~cm}$ plane is only $13 \%$ of that in the plane of the point source while the value is $64 \%$ with simple back projection.

An N, S and L letter phantom (see Koral et al. 1977) is reconstructed by a thinned-array ART2 algorithm with $r=1 \cdot 0$. A 2\% stopping criterion was satisfied after 4, 5, 6 and 7 iterations in 1,36,43 and 1 of the 81 subsets respectively. Values for the strength in each letter (table 2) are obtained by

Table 2. Comparison of actual and reconstructed strength, plane-by-plane and total

(a) Letter phantom with $2 \%$ stopping criterion

\begin{tabular}{cccc}
\hline Letter & $\begin{array}{c}\text { Plane } \\
\text { depth } \\
(\mathrm{cm})\end{array}$ & $\begin{array}{c}\text { Reconstructed } \\
\text { values }\end{array}$ & Correct values \\
\hline $\mathrm{N}$ & $2 \cdot 2$ & $2.51 \mu \mathrm{Ci}(43.2 \%)$ & $3.60 \mu \mathrm{Ci}(48.0 \%)$ \\
$\mathrm{S}$ & 3.3 & $1.77 \mu \mathrm{Ci}(30.5 \%)$ & $2 \cdot 30 \mu \mathrm{Ci}(30.6 \%)$ \\
$\mathrm{L}$ & 4.3 & $1.52 \mu \mathrm{Ci}(26.2 \%)$ & $1.60 \mu \mathrm{Ci}(21.3 \%)$ \\
Total & & $5.80 \mu \mathrm{Ci}(100 \%)$ & $7.50 \mu \mathrm{Ci}(100 \%)$ \\
\hline
\end{tabular}

(b) Volume phantom after four iterations

\begin{tabular}{|c|c|c|c|c|}
\hline \multirow{2}{*}{$\begin{array}{l}\text { Plane } \\
\text { depth } \\
\text { (cm) }\end{array}$} & \multicolumn{3}{|c|}{ Reconstructed values } & \multirow[b]{2}{*}{ Correct values } \\
\hline & $\begin{array}{c}\text { ART } \\
r=1\end{array}$ & $\begin{array}{l}\text { ART2 } \\
r=1\end{array}$ & $\begin{array}{c}\mathrm{ART} \\
r=1 / 8\end{array}$ & \\
\hline $2 \cdot 2$ & $10.7 u \mathrm{Ci}(17.7 \%)$ & $10 \cdot 1 \mu \mathrm{Ci}(17.0 \%)$ & $6.4 \mu \mathrm{Ci}(13.3 \%)$ & $18.8 \mu \mathrm{Ci}(13.0 \%)$ \\
\hline $2 \cdot 6$ & $12.2 \mu \mathrm{Ci}(20.1 \%)$ & $11 \cdot 3 \mu \mathrm{Ci}(18.9 \%)$ & $9.0 \mu \mathrm{Ci}(18.5 \%)$ & $27.0 \mu \mathrm{Ci}(18.6 \%)$ \\
\hline $3 \cdot 3$ & $13.6 \mathrm{uCi}(22.5 \%)$ & $13 \cdot 1 \mu \mathrm{Ci}(22 \cdot 1 \%)$ & $11.9 \mu \mathrm{Ci}(24.4 \%)$ & $35.9 u \mathrm{Ci}(\mathbf{2 4 . 8} \%)$ \\
\hline $4 \cdot 3$ & $24.0 \mu \mathrm{Ci}(39 \cdot 7 \%)$ & $25.0 \mu \mathrm{Ci}(42.0 \%)$ & $21.4 \mu \mathrm{Ci}(43.8 \%)$ & $63.0 \mu \mathrm{Ci}(43.5 \%)$ \\
\hline Total & $60.5 \mu \mathrm{Ci}(100 \%)$ & $59.6 \mu \mathrm{Ci}(100 \%)$ & $48.8 \mu \mathrm{Ci}(100 \%)$ & $144 \cdot 7 \mu \mathrm{Ci}(100 \%)$ \\
\hline
\end{tabular}

defining the region occupied and multiplying the average reconstructed image-strength density in microcuries per image element times the total area in image elements. The general tencency for the reconstructed strengths to be about $25 \%$ low compared to the true values is attributable to neglecting the Anger camera photopeak efficiency. The values for the strength of each letter as a percentage of the total strength agree with the true values within the accuracy of the measurement.

A more difficult phantom to reconstruct is one in which the radioactivity is continuously distributed throughout a volume. Such a phantom is a cylinder $5 \mathrm{~cm}$ in diameter and $3.5 \mathrm{~cm}$ long which contains both a $2 \mathrm{~cm}$ diameter cold cylinder at the bottom and a smaller cold cylinder near the top, as sketched 
in fig. 5. The results for this phantom, as viewed from the top, are shown at the right of the figure where reconstructions from the thinned-array ART algorithm with relaxation parameter equal to 1 and $1 / 8$ are compared. It is seen that the 'under-relaxed' result $(r<1)$ is superior as the small defect is rounder in the $2 \cdot 65 \mathrm{~cm}$ plane and the strength is more uniform in the front and back planes.

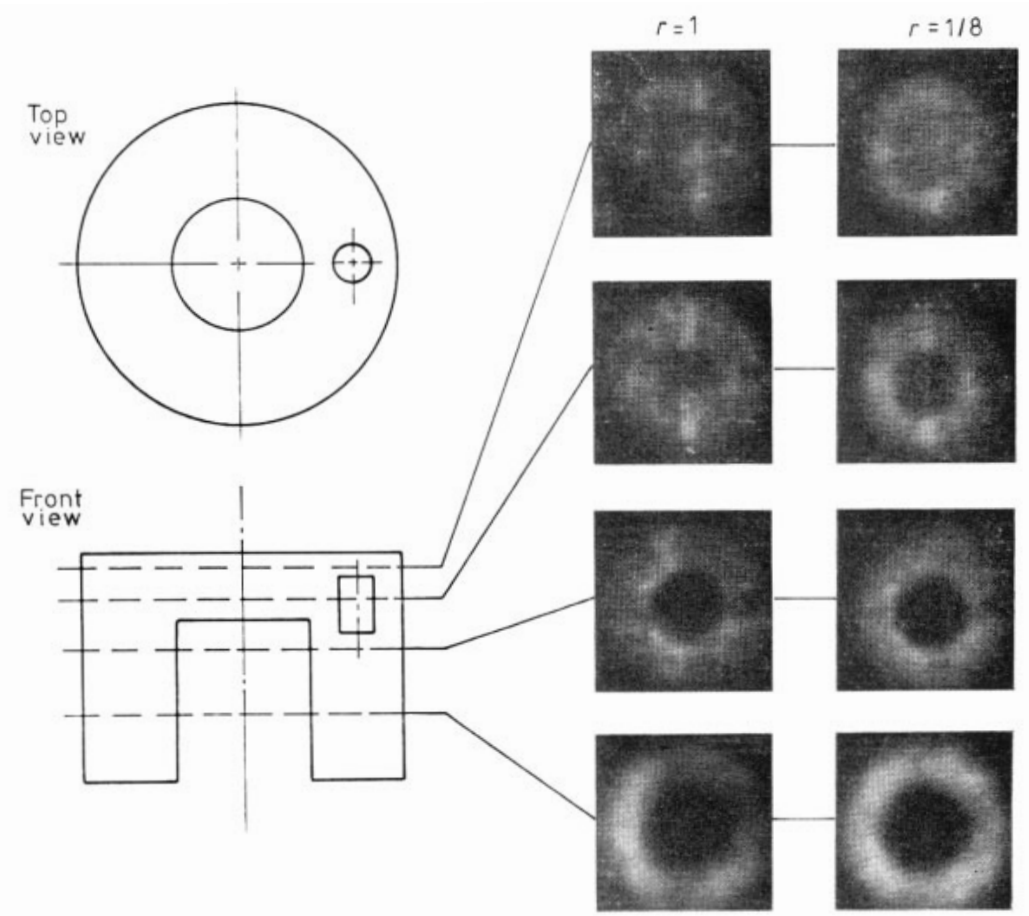

Fig. 5. Four-iteration ART reconstructions of a $5 \mathrm{~cm}$ diameter volume phantom. The mean depths of the planes intersect the phantom as shown and correspond to $k=7,6,5$ and 4 . The images are quite faithful, within the limits of degraded depth resolution, especially with $r=1 / 8$.

Both reconstructions show elongation of the cold cylinders, the result of the limited angular range of the projections, and both resolve the $6 \mathrm{~mm}$ diameter, $8 \mathrm{~mm}$ long cold defect. There is an artifactual cross in the front planes, especially with $r=1$, due to the effective camera mask being too large.

Reconstructed strengths for the four planes after 4 iterations are given in table 2 as a function of algorithm, as well as the actual values calculated from the geometrical volumes. The reconstructed values are in greater disagreement with the true values in this case than in the case of the letter phantom. Assuming a photopeak efficiency of $75 \%$, one finds a discrepancy in the total strength with $r=1$ of $51 \mu \mathrm{Ci}$. Selfabsorption accounts for only $46 \%$ of this discrepancy. With the relaxtion parameter equal to 1 , table 2 shows little difference between employing ART or ART2 (a fact which is also true of the images qualitatively). The ART results with $r=1 / 8$ produce smaller values than those with $r=1$, indicating that 4 iterations are not sufficient for this algorithm to converge 
quantitatively although the image is not visually changed with further iterations. The percentage distribution among planes with $r=1 / 8$, however, is better than with $r=1$ and is actually surprisingly good since an absorption correction has not been made.

For the above phantom, fig. 6(a) shows a comparison of profiles through the cold spot in the $2 \cdot 65 \mathrm{~cm}$ plane with ART reconstruction $(r=1 / 8)$ and with simple back-projection of the corrected correlation coefficients. The superiority of the ART technique is seen in the clear shoulder located over the cold defect

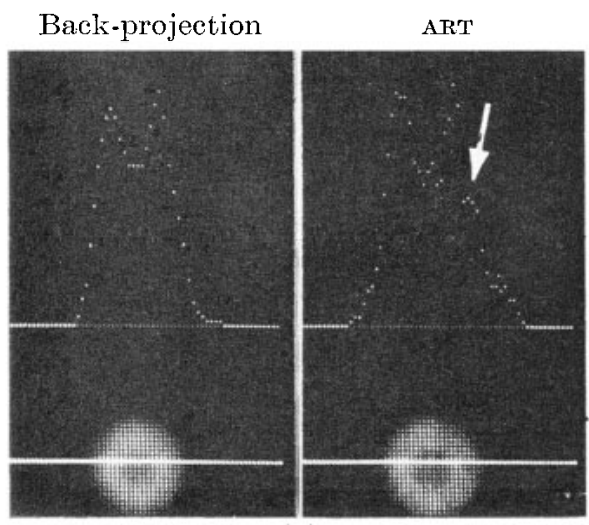

(a)
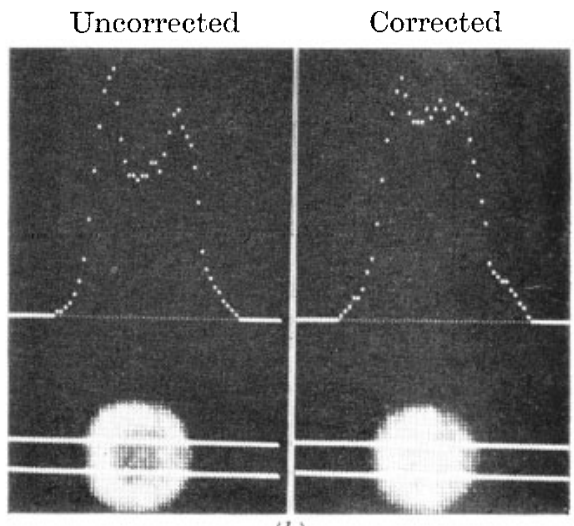

(b)

Fig. 6. Results from the volume phantom. (a) Profile comparison ( $k=6$ planc). The arrow marks the location of the $6 \mathrm{~mm}$ diameter cold defect which is seen with ART but not with back-projection. (b) Profiles correspond to the band across the image $(k=7$ plane). With correction the dip in the middle of what should be a disc disappears.

compared to the unperturbed profile obtained with back-projection. The effect of correcting the correlation coefficients (eqn (3)) was investigated by reconstructing a set of images without the correction. A large qualitative change occurs in the near plane as shown by the normalised profiles of fig. $6(b)$. Without the correction, large-angle rays which pass through the centre of the disc are sufficiently reduced from their correct values so as to combine with normally incident rays that are small in value due to the large cold defect, to produce an incorrect dip in the strength. With the correction, a much more uniform profile is produced. The quantitative result of the correction is an increase in calculated strength by a factor of approximately $1 \cdot 5$.

The speed of convergence of the iterative process has been a difficult parameter to assess in this study. Qualitatively, all algorithms have converged rapidly with 1 or at most 2 iterations revealing the image in very nearly its final form. Quantitative values continue to change for iterations beyond 2, especially in the under-relaxed case. 'This behavior is illustrated in fig. 7 for one subset of data in the case of reconstruction of the cylindrical phantom. The variance satisfies a $2 \%$ stopping criterion after 5 iterations when $r=1$, but only after 16 iterations when $r=1 / 8$. Three iterations are sufficient to obtain a $1 \%$ total-strength stability when $r=1$ while 7 are necessary to meet this criterion when $r=1 / 8$. 


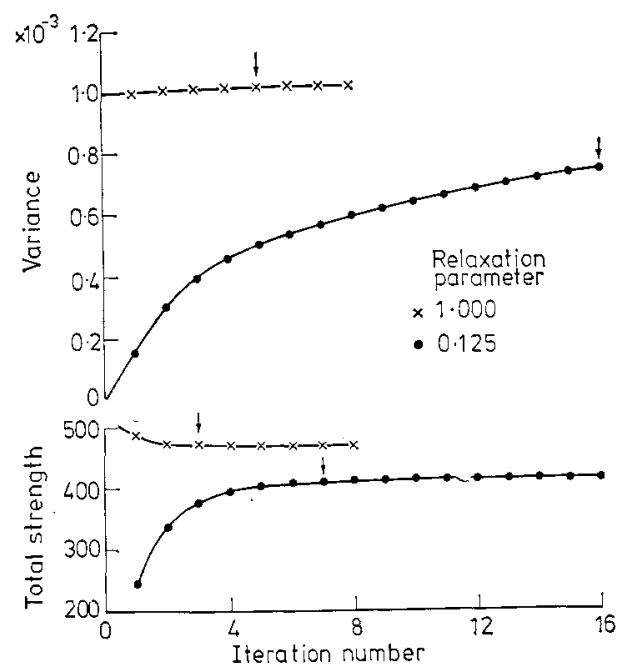

Fig. 7. Speed of convergence for one subset of data in the case of the volume phantom. Total strength, relative units and variance from the mean are plotted against number of iterations. For this single subset, final total strength appears $15 \%$ higher with $r=1$ compared to $r=1 / 8$.

\section{Conclusions}

From the previous results one can conclude the following: Projections in a conical volume over a limited angular range produce images which are interpretable, but which feature a resolution in the direction normal to the detector plane which is inferior to that in planes parallel to the detector. Two-dimensional simulations show that the effect upon the image of array thinning to save computer core space is small while the effect of under-relaxation is to produce an improvement for noisy data. Experimental measurements upon small phantoms confirm the beneficial effects of relaxation upon image quality and the superiority of the ART algorithm compared to non-iterative back-projection. Finally, although the absolute strength for a volume phantom is low by a factor which is greater than one would expect based on an assumed camera photopeak efficiency of $75 \%$, relative strength values are good, even though absorption has been ignored.

\section{APPENDIX 1}

We wish to derive the relationship between the time-correlation coefficient and the emitting strength of an image element for the general three-dimensional finite-geometry case. The starting point is the result for a single detector and a number of discrete sources (Koral et al. 1975):

$$
Q_{i}=(p-q) N_{i} \Omega_{i}
$$

where $Q_{i}$ is the mean-subtracted correlation coefficient for the $i$ th region of the aperture, $p$ is the peak and $q$ the side-lobe value of the autocorrelation of the code, $N_{i}$ is the mean number of gamma rays emitted per time interval for the $i$ th 
source, and $\Omega_{i}$ is the solid angle of the detector as seen from the $i$ th source. (Note that in the reference all sources were taken to see a solid angle of 1 so that $\Omega_{i}$ did not appear explicitly.)

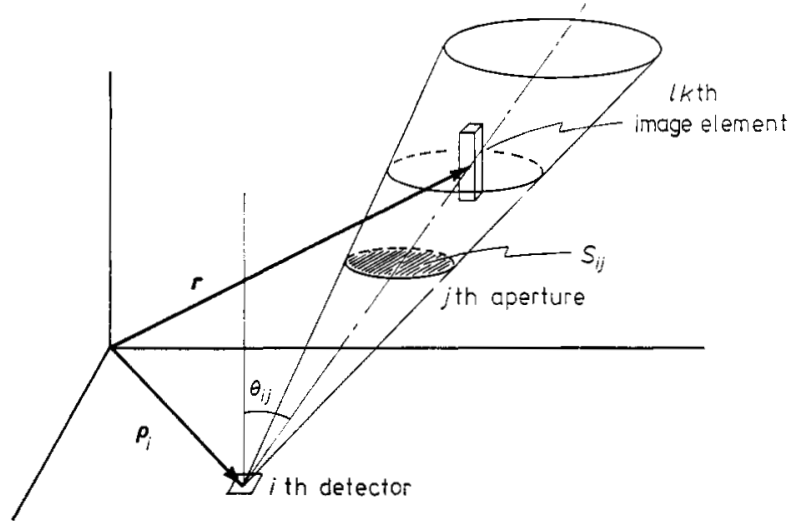

Fig. A1. Sketch of image volume associated with $i j$ th correlation coefficient and position of $l k$ th image element within that volume.

We now generalise the situation to that shown in fig. Al with $n(\mathbf{r})$ equal to the source strength per unit volume:

$$
Q(\boldsymbol{p}, \boldsymbol{r})=(p-q) \iint n(\boldsymbol{r}) \mathrm{d}^{2} \rho \cos \theta / u^{2}(\boldsymbol{r}, \boldsymbol{p}) \mathrm{d}^{3} r
$$

where

$$
\boldsymbol{u}=\boldsymbol{r}-\rho \quad \text { and } \quad u^{2}(\boldsymbol{r}, \rho)=\boldsymbol{u} \cdot \boldsymbol{u} .
$$

Assuming that the detectors are quite small and of area $D$,

$$
Q_{i}(\boldsymbol{p}, \boldsymbol{r})=(p-q) D \int \mathrm{d}^{3} r n(\boldsymbol{r}) \cos \theta_{i} / u^{2}\left(\boldsymbol{r}, \boldsymbol{\rho}_{i}\right), \quad i=1,2, \ldots, N_{\mathrm{D}}
$$

where $\rho_{i}$ is the vector to the centre of the $i$ th detector element and $N_{D}$ is the number of such elements. Then specify that the image volume, $V_{i j}$, to be associated with the particular correlation coefficient, $Q_{i j}$ is that enclosed by a cone with apex at $\rho_{i}$ and with sides specified by the open area $S_{i j}$ of the $j$ th aperture as seen from the $i$ th detector (fig. Al).

$$
Q_{i j}=(p-q) D \int_{V_{i j}} \mathrm{~d}^{3} r n(\boldsymbol{r}) \cos \theta_{i} / \iota^{2}\left(\boldsymbol{r}, \boldsymbol{\rho}_{i}\right), \quad j=1,2, \ldots, N_{\mathrm{A}}
$$

Now consider $N_{\mathrm{p}}$ discrete depths indexed by $k$ and make the further assumption that the image distribution is changing slowly enough so that we can sum the volume of a truncated cone at each of the discrete depths times the sourcestrength density at a single central location within the cone to evaluate the integral above. If we choose, in particular, the source-strength density along the $i j$ th ray, $n_{l k}$, where $l$ is a function of $i, j$ and $k$, then

$$
Q_{i j}=(p-q) D \sum_{k=k_{i}}^{k_{f}} n_{l k} \cos \theta_{i j} / u^{2}\left(\boldsymbol{r}_{l, k}, \boldsymbol{\rho}_{i}\right) \int_{V_{l k}} \mathrm{~d}^{3} r
$$


The volume integral will be approximated by the area $S_{i j}$ back-projected into the plane at depth $A_{k}$ times the thickness of the image elements in that plane, $t_{k}$, so

$$
\int_{V i, k} \mathrm{~d}^{3} r=S_{i j}\left(A_{k}+B\right)^{2} t_{k} / B^{2}
$$

where $B$ is the distance from the aperture to the detector. Therefore,

$$
Q_{i j}=(p-q) D \cos ^{3} \theta_{i j} S_{i j} B^{-2} \sum_{k=k_{i}}^{k_{f}} n_{l k} t_{k}
$$

The factor outside the summation is a function of only $i$ and $j$ and each correlation coefficient may be corrected by it (eqn (3)) with $F=D(p-q) / B^{2}$.

Finally, if we redefine the image in terms of emission strength per unit area, $I_{l k} \equiv n_{l k} t_{k}$, then

$$
\tilde{Q}_{i j}=\sum_{k=k_{i}}^{k j} I_{l k}, \quad l=f(i, j, k) .
$$

\section{RÉSUMí}

Application de la Technique de Reconstruction Algébrique (ART) à la tomographie à émission à temps codé

Les dispositifs de tomographie à émission à photon simple prennent couramment des projections soit dans un plan sur une gamme angulaire totale ou dans un volume sur une gamme angulaire limitée. L'ouverture à temps codé plane, pseudo-hazard, en conjonction avec une caméra Anger, est un dispositif du type à gamme angulaire limitée. Il utilise des sténopés qui ont une transmission variant en fonction du temps. Précédemment, la reconstruction d'image était effectuée par simple projection des coéfficients obtenus dans le temps en mettant en corrélation la trans. mission des sténopés avec le taux de comptage de l'élément détecteur, ce qui résultait en une image à contraste faible. La Technique de Reconstruction Algébrique est utilisée, et une méthode est introduite, pour la division des coéfficients de corrélation en sous-ensembles permettant d'effectuer la reconstruction en trois dimensions sur mini-ordinateur. Les résultats de simulations et de données expérimentales fantômes montrent que la ART améliore la résolution en profondeur par rapport à la projection, que la sous-relaxation produit de meilleures images dans le cas de données bruyantes, et que la division des coéfficients en sous-ensembles n'a aucun effet sur la qualité. Les images montrent la dégradation attendue de résolution dans la direction perpendiculaire au plan du détecteur dûe à la gamme angulaire limitée des projections, mais donnent des résultats quantitatifs dont les valeurs relatives sont bonnes, bien même que l'atténuation soit négligée.

\section{ZUSAMMENFASSUNG}

Die Anwendung von Algebraisches Abblidrekonstruktionsverfahren (ART) auf zeitkodierte Emissionstomographie

Bei den tomographischen Geräten mit Einzelphotonenemission werden gegenwärtig Projektionen entweder in einer Ebene innerhalb eines vollständigen Winkelbereichs oder in einem Raum bzw. Volumen mit begrenztem Winkelbereich erfasst. Die ebene zeitkodierte Blende mit Pseudo-Willkürlichkeit stellt im Zusammenhang mit einer Anger-Kamera eine Vorrichtung dar, die zum Typus mit beschränktem Winkelbereich gehört. Hierbei werden mehrere Feinlöcher mit einer zeitvariablen Transmission verwendet. Zuvor erfolgte die Abbildrekonstruktion durch einfache Rückprojektion von Koeffizienten, die durch zeitkorrelierende Feinlochübertragung bei Zählraten, die für Detektorelemente zutreffen. Die sich daraus ergebende Abbildung war kontrastarm. Es wird nunmehr das Algebraische Rekonstruktionsverfahren eingesetzt und eine Methode zur Untergliederung der Korrelationskoeffizienten in Untergruppen eingeführt, die die dreidimensionale Rekonstruktion mit Hilfe eines Yinikomputers ermöglicht. Die durch Simulationsversuche und an experimentellen Phantomen erhaltenen Werte zeigen, dass das Algebraische Rekonstruktionsverfahren gegenüber der Rückprojektion zu beserren Bildauflösungen bzw. 
-schärfen führt, dass eine Unterrelaxation bei geräuschbehafteten Daten bessere Abbildungen ergibt und dass die Unterteilung der Korrelationskoeffizienten in Untergruppen keine Auswirkung auf die Qualität der Abbildung hat. Die Abbildungen zeigen die erwartete Schwächung der Auflösung in der Richtung quer zur Detektorebene als Folge des begrenzten Winkelbereichs der Projektionen. Allerdings zeitigen die Abbildungen quantitative Werte von relativ guter Aussage. kraft, obwohl die Schwächung der Schärfe ausser Acht gelassen worden ist.

\section{REFERENCES}

Budirger, T. F., and Gullberg, G. T., 1974, IEEE Trans. Nucl. Sci., NS-21 (3), $2-20$.

Colsher, J. D., 1976, Ph.D. Thesis, University of Pennsylvania. (Also as UCRL-52170, Lawrence Livermore Laboratory, University of California, Livermore, California).

Herman, G. T., and Lest, A., 1976, Comput. Biol. Med., 6, 273-294.

Herman, G. T., Lent, A., and Rowland, S. W., 1973, J. Theor. Biol., 42, 1-32.

Keyes, J. W., JR., Orlandea, N., Hemtderks, W. J., Leonard, P. F., and Rogers, W. L., 1977, J. Nucl. Med, 18, 381-387.

Korat, K. F., Freitas, J. E., Rogers, W. L., and Keyes, J. W., Jr., 1979, J. Nucl. Med., 20, 345-349.

Koral, K. F., Krold, G. F., and Rogers, W. L., 1977, in Proc. Vth Int. Conf. on Informa. tion Processing in Medical Imaging (Nashville: ORNL) pp. 252-265.

Koral, K. F., Rogers, W. L., and Kroll, G. F., 1975, J. Nucl. Med., 16, 402-413.

KUHL, D. E., Edwards, R. Q., Ricci, A. R., YACOB, R. J., MICH, T. J., and AIAvi, A., 1976, Radiology, 121, 405-13.

Vogel, R. A., KIrch, D. L., LeFree, M. T., Kinngensmith, W. C., Trow, R. S., and Steele, P. P., 1977, in Proc. Vth Int. Conf. on Information Processing in Medical Imaging (Nashville: ORNL) pp. 636-646.

Vogel, R. A., KIRCH, D., LeFree, M., and Steele, P., 1978, J. Nucl. Med., 19, 648-654. 